\title{
FAMILY CENTRED CARE - FROM A SYSTEMS PERSPECTIVE \\ B. Westrup
}

Neonatology, Karolinska University Hospital, Stockholm, Sweden

Family centred care has gained an increasing interest in the field of peadiatrics and has become widely implemented. However, it is still a hot topic in neonatology and by many not considered as important for families of newborn infants. This lecture will address the scientific context/background, its role from a systems perspective and the scientific evidence of family centred care in regards to morbidity and long-term development for newborn population. Future developments will also be discussed. 